\title{
The Effects of Pharmacological Opioid Blockade on Neural Measures of Drug Cue-Reactivity in Humans
}

\author{
Kelly E Courtney', Dara G Ghahremani ${ }^{2}$ and Lara A Ray, ${ }^{*, 1,2}$ \\ 'Department of Psychology, University of California, Los Angeles, CA, USA; ${ }^{2}$ Department of Psychiatry and Biobehavioral Sciences, University of \\ California, Los Angeles, CA, USA
}

\begin{abstract}
Interactions between dopaminergic and opioidergic systems have been implicated in the reinforcing properties of drugs of abuse. The present study investigated the effects of opioid blockade, via naltrexone, on functional magnetic resonance imaging (fMRI) measures during methamphetamine cue-reactivity to elucidate the role of endogenous opioids in the neural systems underlying drug craving. To investigate this question, non-treatment seeking individuals with methamphetamine use disorder ( $N=23 ; 74 \%$ male, mean age $=34.70(S D=8.95)$ ) were recruited for a randomized, placebo controlled, within-subject design and underwent a visual methamphetamine cue-reactivity task during two blood-oxygen-level dependent (BOLD) PMRI sessions following 3 days of naltrexone (50 mg) and matched time for placebo. $\mathrm{FMRI}$ analyses tested naltrexone-induced differences in BOLD activation and functional connectivity during cue processing. The results showed that naltrexone administration reduced cue-reactivity in sensorimotor regions and related to altered functional connectivity of dorsal striatum, ventral tegmental area, and precuneus with frontal, visual, sensory, and motor-related regions. Naltrexone also weakened the associations between subjective craving and precuneus functional connectivity with sensorimotor regions and strengthened the associations between subjective craving and dorsal striatum and precuneus connectivity with frontal regions. In conclusion, this study provides the first evidence that opioidergic blockade alters neural responses to drug cues in humans with methamphetamine addiction and suggests that naltrexone may be reducing drug cue salience by decreasing the involvement of sensorimotor regions and by engaging greater frontal regulation over salience attribution.

Neuropsychopharmacology (2016) 4I, 2872-288I; doi:I0.1038/npp.2016.99; published online I3 July 2016
\end{abstract}

\section{INTRODUCTION}

The incentive salience model posits that addiction is largely caused by progressive neuroadaptation that results in sensitization that follows repeated drug use. This sensitization process is implicated to occur within the dopaminergic pathways that subserve attribution of motivational salience to mental representations of stimuli and actions (eg, drug ingestion), thus rendering these stimuli highly salient and ultimately 'wanted' or craved (Berridge and Robinson, 2003; Robinson and Berridge, 2001). Following sensitization of these pathways, the expression of incentive salience (ie, craving) can be activated by the release of dopamine that is initiated in response to drug cues or priming doses of the drug itself (de Wit, 1996).

Dopaminergic function within the striatum, particularly the dorsal striatum (Everitt and Robbins, 2005), is thought to be critically important for the development of incentive salience or craving for drugs (Volkow et al, 2006; VollstadtKlein et al, 2010); however, functional interactions between

* Correspondence: Dr LA Ray, Department of Psychology, University of California, 1285 Franz Hall, Box 951563, Los Angeles, CA 90095-1563, USA, Tel: +I 310794 5383, Fax: +I 310206 5895,

E-mail: lararay@psych.ucla.edu

Received 20 December 2015; revised 15 May 2016; accepted 8 June 2016; accepted article preview online 17 June 2016 the endogenous opioid and dopaminergic systems have been implicated in the development of sensitization to various drugs of abuse, including stimulants. For example, increased $\mu$-opioid receptor binding in the frontal, temporal, anterior cingulate, and amygdalar cortices, as evidenced by positron emission tomography, is associated with increased subjective cocaine craving in cocaine-dependent men during early withdrawal (Zubieta et al, 1996). Further, increases in preproenkephalin mRNA expression and decreases in $\mu$-opioid receptor levels in the dorsal and ventral striatum were observed in sensitized wild-type mice following 7 days of daily intraperitoneal injections (i.p.) of methamphetamine, while no changes were observed in $\mu$-opioid receptor knockout mice. These latter opioidergic changes were associated with altered dopaminergic function in the same striatal regions (Tien et al, 2007) and highlight potential mechanisms by which the two systems interact to result in the experience of drug craving.

Given the role of endogenous opioids in the neurobiology of addiction, it is crucial to establish how manipulation of the opioid system through opioidergic blockade may disrupt addiction-maintaining processes such as cue-induced craving. In order to provide an efficient blockade of opioid receptors, the present study used naltrexone, a clinically relevant opioid receptor antagonist with greatest affinity for the $\mu$ - and $\kappa$-opioid receptors in humans (Emmerson et al, 
1994; Toll et al, 1998). The efficacy of naltrexone for substance use disorders (eg, Miranda et al, 2014; Monti et al, 1999; Ray et al, 2008; Syed and Keating, 2013), including amphetamine (Itzhak and Ali, 2002; Jayaram-Lindstrom et al, 2008) and methamphetamine (Anggadiredja et al, 2004; Ray et al, 2015a) may be subserved by its attenuation of drug craving. Naltrexone is thought to modulate reinforcementdriven behavior via blocking dopamine release in the mesolimbic dopamine system, primarily acting on the pathway from the ventral tegmental area (VTA) to the ventral striatum (ie, nucleus accumbens (NAcc)) (Benjamin et al, 1993; Lee et al, 2005); however, this theoretical pathway of naltrexone's effects on behavior has not been directly tested in human models of cue-induced craving.

This study employed a novel methamphetamine cueexposure paradigm for functional magnetic resonance imaging (fMRI) and functional connectivity analyses as tools for probing naltrexone-induced modulation of the incentive salience system in a sample of individuals with methamphetamine use disorder. The primary aims of the present study were to determine: (1) Whether naltrexone (50 mg) vs placebo moderates blood-oxygen-level dependent (BOLD) measures of methamphetamine cue processing (ie, cuereactivity) and (2) whether naltrexone's effect on methamphetamine cue-reactivity is associated with differential functional connectivity from the striatum and VTA (regions subserving reinforcement-driven behavior) and the precuneus (a region reliably shown to respond to drug cues; Courtney et al, 2014a; Engelmann et al, 2012; Schacht et al, 2013a) to other brain regions, primarily in the cortex, during methamphetamine cue processing, as compared to placebo. Exploratory aims were to test: (1) The effect of naltrexone $(50 \mathrm{mg})$ on cerebral blood flow (CBF) in this sample with methamphetamine use disorder, as naltrexone-induced $\mathrm{CBF}$ alterations have been observed in alcohol-dependent populations (Catafau et al, 1999) and represent a potential confound for the analyses of the BOLD fMRI signal, and (2) whether subjective reports of cue-induced methamphetamine craving are related to neural markers of methamphetamine cue processing. The overall goal of the study was to elucidate the functional neural pathways by which endogenous opioids may be contributing to the expression of salience to drug cues in humans with a substance use disorder.

\section{MATERIALS AND METHODS}

\section{Study Design}

Twenty-four medically eligible non-treatment-seeking individuals with a methamphetamine use disorder completed a within-subjects protocol that involved the completion of two, 3-day medication administrations (naltrexone/placebo; medication order randomized between subjects) and an MRI scan on the third day (at target dose of naltrexone and matched time for placebo; see SI for further details). Participants received a $25 \mathrm{mg}$ dose on day one and $50 \mathrm{mg}$ doses on days two and three in the naltrexone condition, and colormatched placebo pills in the placebo condition, taken under study staff supervision. Sixteen participants were scanned prior to laboratory testing as part of a 7-day inpatient protocol investigating the effects of naltrexone on subjective response to methamphetamine administration (Ray et al, 2015a) and the remaining eight participants were administered the medication daily on an intensive outpatient basis. Abstinence from alcohol and substances (including methamphetamine) during the two, 3-day study periods was required and verified daily by a Breathalyzer test (Dräger Medical Inc., Telford, PA) and urine toxicology screen. A washout period of at least 7 days between medication conditions was required for both inpatients and outpatients to reduce medication carry-over effects. There were no differences in demographic variables across the inpatient and outpatient subsamples used in the final analysis (independent $t$-tests or Fisher's Exact Tests $p$ s $>0.05$; see Supplementary Table S1) and all recruitment procedures and eligibility criteria were identical across the inpatient and outpatient subsamples.

\section{Neuroimaging Procedures}

Neuroimaging data were acquired using a 3 Tesla Siemens Trio MRI scanner at the UCLA Staglin Center for Cognitive Neuroscience. The protocol began with initial structural scans: T2-weighted, high resolution, matched-bandwidth (MBW; TR, $5 \mathrm{~s}$; TE, $34 \mathrm{~ms}$; FOV, $192 \mathrm{~mm}$; matrix, $128 \times$ 128; sagittal plane; slice thickness, $4 \mathrm{~mm}$; 34 slices) and a magnetization-prepared rapid-acquisition gradient echo scan (MPRAGE; TR, 1.9 s; TE, 2.26 ms; FOV, 250 mm; matrix, $256 \times 256$; sagittal plane; slice thickness, $1 \mathrm{~mm}$; 176 slices). These were followed by two runs of the Methamphetamine Cues Task (slice thickness, $4 \mathrm{~mm} ; 34$ slices; TR, $2 \mathrm{~s}$; TE, $30 \mathrm{~ms}$; flip angle, $90^{\circ}$; matrix, $64 \times 64$; FOV, $192 \mathrm{~mm}$; voxel size, $3 \times 3 \times 4 \mathrm{~mm}^{3}, 150$ functional T2*-weighted EPIs) and a pseudo-continuous arterial spin labeling scan (ASL; slice thickness, $5 \mathrm{~mm}$; TR, $4 \mathrm{~s}$; TE, $22.62 \mathrm{~ms}$; FOV, $220 \mathrm{~mm}$; matrix, $64 \times 64,30$ slices).

The Methamphetamine Cues Task included four blocks of methamphetamine cue pictures and four blocks of control cue pictures, pseudo-randomly presented. Each block consisted of four pictures, presented for $5 \mathrm{~s}$ each, for a total of 32 pictures from each condition. Following each block of pictures, a craving question was presented on the screen prompting participants to rate their current urge to use methamphetamine using the four buttons on a response box corresponding to a four-item urge scale ( $1=$ no urge, $2=$ low urge, $3=$ moderate urge, $4=$ high urge) (see SI for more details).

\section{Data Analysis}

One subject was excluded from all analyses due to excessive motion (exceeding $3 \mathrm{~mm}$ translation) during fMRI data acquisition, leaving a final sample of 23 individuals for all analyses. FSL 5.0 (www.fmrib.ox.ac.uk/fsl) was used for all imaging analyses. ASL and fMRI data were motion corrected, brain extracted, smoothed (FWHM Gaussian kernel $6 \mathrm{~mm} /$ $5 \mathrm{~mm}$, ASL/fMRI respectively), and the fMRI data were highpass filtered using a $100 \mathrm{~s}$ cutoff in the temporal domain. Mean CBF images from the ASL data were created by averaging the simple subtraction of label and control images, according to a one-compartment perfusion model (delay time $1.2 \mathrm{~s}$, label time $1.2 \mathrm{~s}$, label efficiency 0.9 , blood/tissue compartment (lambda) 0.9, T1-blood $1650 \mathrm{~ms}$ ). CBF and EPI images were first registered to the MBW, then to the 
MPRAGE using affine linear transformations, and then into standard space (Montreal Neurological Institute, MNI avg152 template).

The Methamphetamine Cue $>$ Control Cue contrast was specified in the first-level models. Higher-level analyses combined these contrast images within subjects (across runs; fixed effects analyses) and between subjects (within medication conditions and across medication conditions). Z-statistic images were thresholded with cluster-based corrections for multiple comparisons based on the theory of Gaussian Random Fields with a cluster-forming threshold of $Z>2.3$ and a cluster-probability threshold of $p<0.05$ (Worsley, 2001).

The four seed regions of interest (ROIs) used in the functional connectivity analyses were the VTA, ventral and dorsal striatum, and precuneus (see Supplementary Figure S1 for an image of the ROIs). The VTA was anatomically defined using a midbrain probabilistic atlas developed by Murty et al (2014). The ventral striatum was anatomically defined using the bilateral NAcc regions from the HarvardOxford probabilistic atlas. The dorsal striatum was anatomically defined using the bilateral caudate regions from the Harvard-Oxford probabilistic atlas. Given the large size of the caudate region and our intended focus on areas specifically sensitive to drug cues, we further functionally constrained the anatomically defined bilateral ROI by the activation from the Methamphetamine Cue $>$ Control Cue contrast combined across subjects and medication conditions (thresholded at $Z>2.3$, as described above). Similarly, the bilateral precuneus was anatomically defined by the right and left precuneus regions from the Harvard-Oxford probabilistic atlas, and further functionally constrained by the activation from the Methamphetamine Cue $>$ Control Cue contrast due to its large size. Functional constraint of the dorsal striatum and precuneus resulted in slightly asymmetric ROIs (see SI and Supplementary Figure S1).

Functional connectivity was assessed using psychophysiological interaction (PPI) analysis (O'Reilly et al, 2012), which examined coupling of the time series of the different seed ROIs with the rest of the brain with respect to the Methamphetamine Cue $>$ Control Cue contrast. The firstlevel PPI models included four regressors: the main 'psychological' regressor to model the difference in task conditions (Methamphetamine Cues-Control Cues), a second 'psychological' regressor to account for the shared variance between task conditions (Methamphetamine Cues+Control Cues), a 'physiological' regressor to model the seed time course, and a 'psychophysiological interaction' regressor which is the product of the main 'psychological' and 'physiological' regressors. A whole-brain contrast image for each seed PPI was computed from these models and submitted for higherlevel analyses as described above. A reduced cluster-forming threshold of $Z>1.96$ and a cluster-probability threshold of $p<0.05$ was employed as the threshold for the PPI $Z$-statistic images due to the reduction in power inherent in the PPI analyses as compared to the standard whole-brain contrasts. Self-reported methamphetamine craving immediately following the presentation of methamphetamine cues during the task, averaged across medication conditions (ie, tonic craving), was entered as a covariate of interest in separate higher-level analyses paralleling the models described above.

\section{RESULTS}

Twenty-three individuals $(74 \%$ male, mean age $=34.70$ $(\mathrm{SD}=8.95))$ completed the within-subjects scanning protocol and provided useable data for analysis (Supplementary Table S1). All 23 individuals met DSM-IV and DSM-5 criteria for a current (ie, past month) methamphetamine use disorder (DSM-IV dependence/abuse: 20/3; DSM-5 severity: 1 mild, 6 moderate, 16 severe). All participants reported extensive experience smoking methamphetamine.

\section{Effects of Opioid Blockade on CBF}

Mean global gray matter CBF estimates were not found to significantly differ between placebo $(M=51.348)$ and naltrexone $(M=52.564)$ conditions $(t(22)=-0.825, p=0.418)$, and voxel-wise comparison of whole-brain global gray matter $\mathrm{CBF}$ estimates did not reveal significant differences, even at a very low uncorrected threshold of $Z>1.96$. Thus, CBF estimates were not included as covariates in BOLD analyses.

\section{Cue-Induced Craving}

Repeated measures ANOVAs revealed a significant effect of cue type on subjective methamphetamine craving reported during presentation of both methamphetamine and control cues while participants performed the fMRI Methamphetamine Cues Task $(F(1,22)=35.96, p<0.001)$. No significant effects for medication condition or for the interaction of medication condition and cue type were observed $(p s>0.8)$. Planned follow-up tests on cue type across scanning blocks revealed that methamphetamine cues were associated with higher self-reported craving ratings than control cues across the entire scan ( $p s<0.01$; Supplementary Figure S2).

\section{BOLD Measures of Methamphetamine Cue-Reactivity}

The main contrast of interest, Methamphetamine Cue $>$ Control Cue, on the 23 individuals averaged across medications (ie, main effect of task) was associated with the activation of a broad set of regions including mesocorticolimbic areas such as the ventral and dorsal striatum and inferior frontal gyrus. Additional areas of activation were found in the frontal lobe (dorsolateral prefrontal cortex (dlPFC), superior frontal gyrus), parietal lobe (precuneus), thalamus, hippocampus, and occipital areas (Supplementary Table S2A; Figure 1). Similar activation patterns were observed in the analysis of the placebo condition alone (Supplementary Table S2B).

Analyses examining the correlation between self-reported methamphetamine craving and activation within the Methamphetamine Cue $>$ Control Cue contrast, averaged across medication conditions, revealed significant positive correlations between craving and activation of the precuneus and occipital regions (lingual gyrus, intracalcarine cortex) during methamphetamine cue processing (Supplementary Table S3, Supplementary Figure S3).

\section{Effects of Opioid Blockade on Cue Processing}

Contrasting the placebo and naltrexone conditions within the Methamphetamine Cue $>$ Control Cue contrasts revealed 

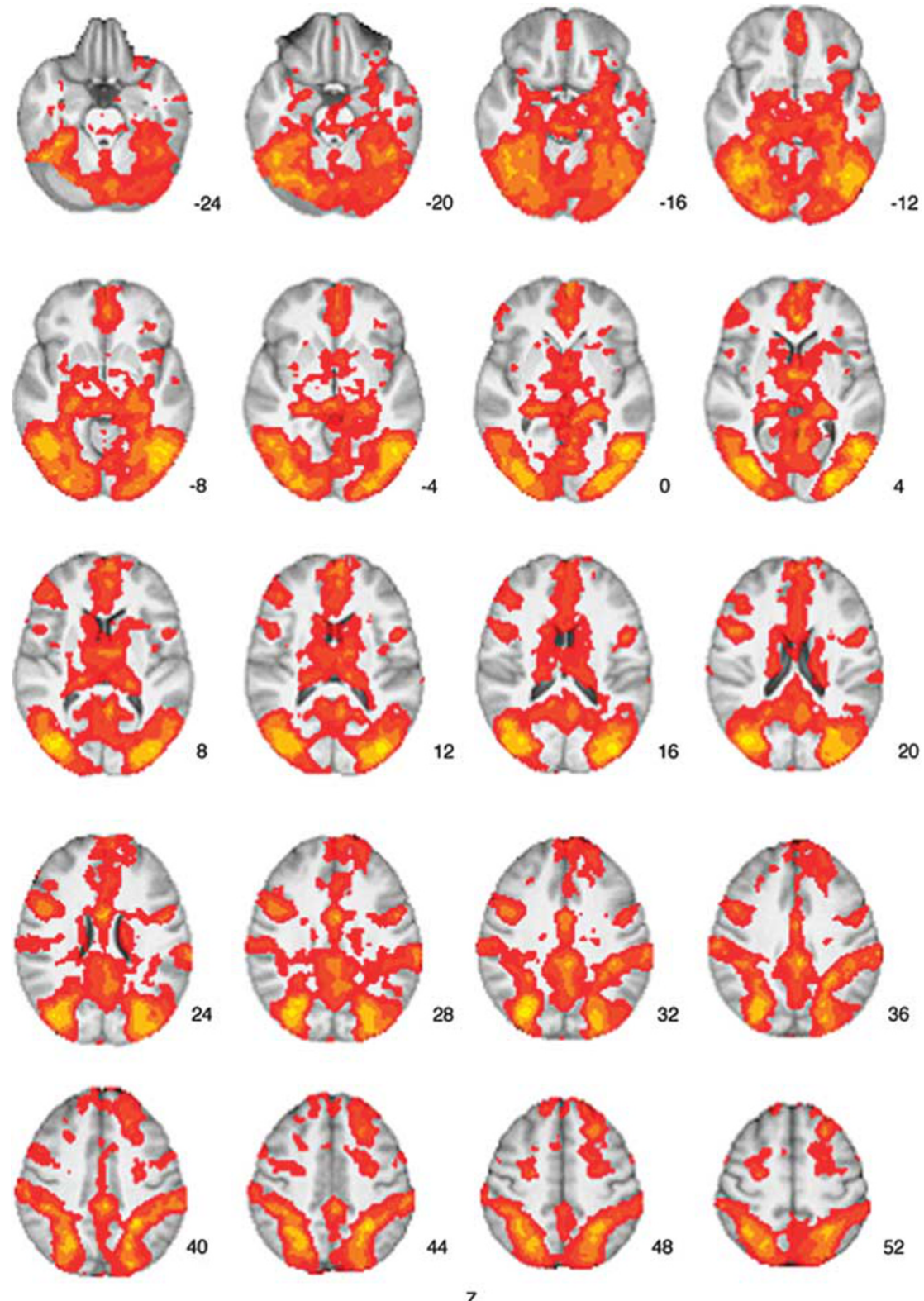

2.30

8.66

Figure I Methamphetamine (MA) Cue > Control Cue activation averaged across medication conditions (see Supplementary Table S2 for list of regions). $Z$-statistic maps are whole-brain cluster-corrected, $Z>2.3, p=0.05$. Coordinates are in $\mathrm{MNI}$ space, and the brain is displayed in radiological convention (left = right).

significantly less activation associated with the naltrexone condition in sensorimotor areas (bilateral precentral and postcentral gyri), and occipital regions (left superior lateral occipital cortex) in the standard whole-brain analysis (Figure 2; Supplementary Table S4).

Functional connectivity within each seed region analysis, with the exception of the ventral striatum (NAcc), was found to differ between medication conditions at a cluster-forming threshold of $Z>1.96$. Specifically, naltrexone was associated with weaker connectivity between the precuneus and frontoparietal regions including the precentral and postcentral gyri (Table 1A, Supplementary Figure S4), stronger connectivity between the VTA and prefrontal, temporal, and parietal regions (Table 1B, Supplementary Figure S5), and stronger connectivity between the caudate and prefronto-occipital regions (Table 1C, Supplementary Figure S6). Effect size maps (in Cohen's $d$ ) for the PPI effects are also presented in the supplementary materials (Supplementary Figures S7-S9).

\section{Craving Related Effects of Opioid Blockade on Functional Connectivity}

The effects of medication on dorsal striatum (caudate) and precuneus functional connectivity was found to correlate with average self-reported craving during cue processing (Methamphetamine Cue $>$ Control Cue; $Z>1.96$ ). Specifically, naltrexone was associated with stronger positive 


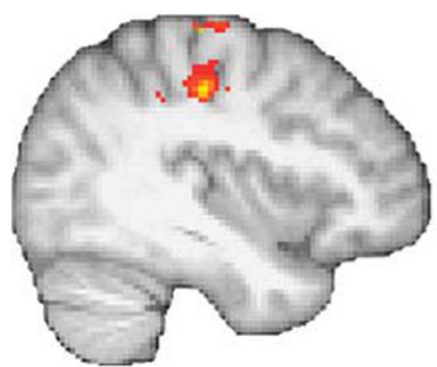

$X=-38$

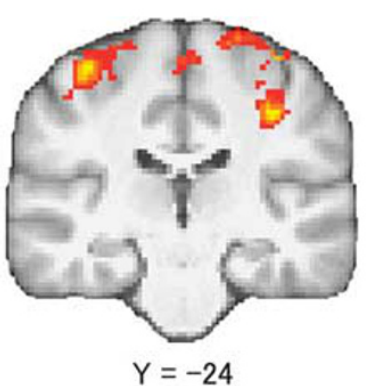

Z

2.30

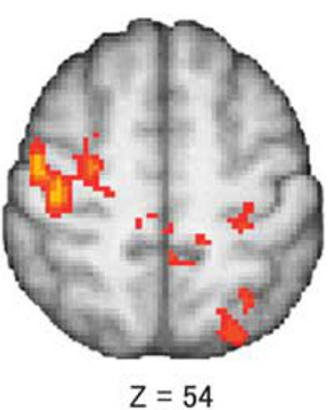

$Z=54$

Figure 2 Methamphetamine (MA) Cue>Control Cue activation that is moderated by medication (placebo (PLAC) > naltrexone (NTX); see Supplementary Table S3 for list of regions). Z-statistic maps are whole-brain cluster-corrected, $Z>2.3, p=0.05$. Coordinates are in MNI space, and the brain is displayed in radiological convention (left $=$ right).

correlations between subjective craving and functional connectivity of the caudate with prefrontal regions during methamphetamine cue processing (Table 2, Supplementary Figure S10). Naltrexone was also associated with weaker positive correlations between self-reported craving and functional connectivity of the precuneus with parietal regions and stronger positive correlations between selfreported craving and functional connectivity of the precuneus with frontal regions during methamphetamine cue processing, as compared to placebo (Table 3, Supplementary Figure S11). In other words, as compared to placebo, naltrexone administration enhanced caudate and precuneus functional connectivity to frontal regions and diminished precuneus connectivity to parietal regions in subjects who endorsed heightened tonic craving. No correlations with craving were observed for the medication effects on VTA functional connectivity.

\section{DISCUSSION}

This study investigated the neural correlates of endogenous opioid blockade, via naltrexone, on methamphetamine cuereactivity in a sample of non-treatment-seeking individuals with a methamphetamine use disorder. fMRI cue-reactivity paradigms represent an ideal platform to probe the involvement of neurobiological pathways subserving the incentive salience system in addiction (Courtney et al, 2016; Ray et al, in press). Given that BOLD measures of drug cue-reactivity have been shown to predict relapse propensity in treatment seeking patients (eg, Beck et al, 2012; Kosten et al, 2006; Schacht et al, 2013b), the investigation of neurochemical systems that alter BOLD measures of cue-reactivity has the potential to improve real-world clinical outcomes in addiction.

\section{BOLD Measures of Methamphetamine Cue-Reactivity}

We developed a novel pictorial methamphetamine cuereactivity paradigm that was designed to elicit methamphetamine craving in individuals with methamphetamine use disorders. The increases in self-reported, subjective methamphetamine craving during the presentation of methamphetamine as compared to control cues highlight the initial efficacy of the task in eliciting cue-induced craving. The primary BOLD results depicted greater methamphetamine ( $v s$ control) cue-elicited activation in regions that are commonly seen to be cue-reactive for other substances in dependent populations (eg, middle frontal gyrus, ACC, posterior cingulate cortex (PCC)/precuneus, thalamus, insula, inferior occipital cortex, and brain stem) (Engelmann et al, 2012; Schacht et al, 2013a), and in commonly described 'reward'- or 'reinforcement'-related regions (eg, left ventral striatum (NAcc), bilateral dorsal striatum (caudate, putamen), VTA, hippocampus, and amygdala). These latter regions primarily rely on dopamine, GABA, opioid, and glutamate signaling and are implicated in the development of incentive salience (Kalivas and Volkow, 2005). Further, we found that greater tonic subjective methamphetamine craving was associated with greater activation of the precuneus, a region highlighted by multiple meta-analyses to be reliably involved in cue-reactivity for various substances of abuse (Engelmann et al, 2012; Schacht et al, 2013a). Taken together, these results suggest that this novel paradigm is an effective probe of methamphetamine cueinduced craving in individuals with methamphetamine use disorders.

\section{Effects of Opioid Blockade on Cue Processing}

Naltrexone was found to modulate cue-reactivity such that naltrexone treatment was associated with attenuated activation in sensorimotor (ie, precentral and postcentral gyri) and visual regions during methamphetamine-cue processing, as compared to placebo. Of the two studies that have directly compared naltrexone to placebo effects on wholebrain BOLD measures of drug cue-reactivity in alcohol- and nicotine-dependent samples, both reported naltrexoneinduced changes in the precentral and postcentral gyri (Lukas et al, 2013; Ray et al, 2015b), suggestive of the reliability of the naltrexone findings in cue-reactivity paradigms across substances of abuse. Importantly, these observed naltrexone-induced effects were independent of global changes to CBF, which had not been ascertained in previous studies, ruling out the possibility that our BOLD effects were confounded by medication-induced changes in CBF. 
Table I Locations Showing a Moderation Effect of Medication (Placebo (PLAC), Naltrexone (NTX)) on (A) Precuneus, (B) Ventral Tegmental Area (VTA), and (C) Caudate Functional Connectivity within the Methamphetamine Cue > Control Cue Contrast

\begin{tabular}{|c|c|c|c|c|c|c|}
\hline Cluster/Region & Hemi & Cluster voxels & $\mathbf{Z}$ & $\mathbf{x}$ & y & $\mathbf{z}$ \\
\hline \multicolumn{7}{|l|}{ (A) Precuneus PPI_PLAC $>$ NTX } \\
\hline Cluster I: Frontoparietal regions & & 1226 & & & & \\
\hline Superior parietal lobule & $\mathrm{L}$ & & 3.37 & -22 & -46 & 48 \\
\hline Postcentral gyrus & $\mathrm{L}$ & & 3.26 & -24 & -28 & 64 \\
\hline Precentral gyrus & L & & 2.84 & -10 & -26 & 46 \\
\hline Cluster I: Prefrontal cortex & & 1679 & & & & \\
\hline Ventromedial prefrontal cortex/caudate (R) & L & & 3.21 & -8 & 54 & -6 \\
\hline Paracingulate gyrus & $\mathrm{L}$ & & 3.18 & -12 & 28 & 26 \\
\hline Ventrolateral prefrontal cortex & L & & 3.13 & -24 & 48 & 2 \\
\hline Parietal operculum cortex & $\mathrm{R}$ & & 2.97 & 52 & -34 & 26 \\
\hline Posterior supramarginal gyrus & $\mathrm{R}$ & & 2.85 & 62 & -40 & 26 \\
\hline \multicolumn{7}{|l|}{ (C) Caudate PPI-NTX $>$ PLAC } \\
\hline Cluster I: Occipital cortex & & 2195 & & & & \\
\hline Occipital pole & $\mathrm{R}$ & & 3.52 & 8 & -92 & 12 \\
\hline Supracalcarine cortex & $\mathrm{R}$ & & 3.04 & 2 & -78 & 18 \\
\hline Cerebellum & $L$ & & 3.03 & -14 & -66 & -24 \\
\hline Cluster 2: Prefrontal cortex & & $|05|$ & & & & \\
\hline Anterior cingulate cortex & $\mathrm{L}$ & & 3.58 & -8 & 34 & 18 \\
\hline
\end{tabular}

Results are from the psychophysiological interaction (PPI) analysis for each seed region (see Methods).

Analyses were whole-brain cluster-corrected at $Z>1.96, p<0.05$.

Note: $\mathrm{X}, \mathrm{Y}$, and $Z \mathrm{MNI}$ coordinates indicate the location of peak voxel activation (or local maxima for subregions) within each cluster. R, right, L, left.

Table 2 Locations of Dorsal Striatal Functional Connectivity (Caudate) that Exhibited Stronger Relationships with Subjective Craving under Naltrexone vs Placebo (NTX>PLAC)

\begin{tabular}{lcccccc}
\hline Region & Hemi & Cluster voxels & $\mathbf{Z}$ & $\mathbf{x}$ & $\mathbf{y}$ & $\mathbf{z}$ \\
\hline $\begin{array}{l}\text { Caudate PPI and MA Craving } \\
\text { Cluster I: Prefrontal regions }\end{array}$ & NTX PLAC & 1053 & & & & \\
$\begin{array}{l}\text { Precentral gyrus } \\
\text { Middle frontal gyrus }\end{array}$ & $\mathrm{L}$ & & 3.49 & -34 & 8 & 30 \\
Inferior frontal gyrus & $\mathrm{L}$ & 3.42 & -46 & 14 & 32 \\
& & 3.28 & -38 & 14 & 26 \\
\hline
\end{tabular}

Results are from the psychophysiological interaction (PPI) analysis showing functional connectivity during methamphetamine cues processing

(Methamphetamine Cue > Control Cue contrast), using the caudate as a functionally constrained, anatomically defined region of interest (see Methods). Analyses were whole-brain cluster-corrected at $Z>1.96, p<0.05$.

Note: $X, Y$, and $Z M N I$ coordinates indicate the location of peak voxel activation (or local maxima for subregions) within each cluster. R, right, L, left.

\section{Effects of Opioid Blockade on Functional Connectivity}

Naltrexone-induced differences in functional connectivity during methamphetamine cue-processing were observed with a number of the a priori seed regions (ie, VTA, precuneus, and caudate, but not NAcc). Specifically, naltrexone was associated with stronger VTA functional connectivity to prefrontal, dorsal striatal and occipitoparietal regions, stronger caudate functional connectivity to prefrontal and occipital regions, and weaker precuneus functional connectivity to sensorimotor regions, as compared to placebo (see Figure 3 for a schematic of the functional connectivity results).

The pharmacological mechanisms subserving the observed functional alterations between the VTA and caudate systems under opioid blockade remain unknown; however, it is plausible that naltrexone may be operating within these regions to increase dopaminergic input to the PFC through the mesocortical pathway (Carr and Sesack, 2000), via 
Table 3 Locations of Precuneus Functional Connectivity that Exhibited Stronger Relationships with Subjective Craving under (A) Placebo vs Naltrexone (PLAC > NTX) and (B) Naltrexone vs Placebo (NTX > PLAC)

\begin{tabular}{|c|c|c|c|c|c|c|}
\hline Cluster/region & Hemi & $\begin{array}{l}\text { Cluster } \\
\text { voxels }\end{array}$ & $\mathbf{Z}$ & $\mathbf{x}$ & y & $\mathbf{z}$ \\
\hline \multicolumn{7}{|c|}{ (A) Precuneus PPI and MA Craving: MA Cue $>$ Control Cue - PLAC > NTX } \\
\hline Cluster I: Parietal cortex & & 991 & & & & \\
\hline Superior parietal lobule & $\mathrm{R}$ & & 3.78 & 36 & -40 & 66 \\
\hline Postcentral gyrus & $\mathrm{R}$ & & 3.32 & 48 & -32 & 58 \\
\hline \multicolumn{7}{|c|}{ (B) Precuneus PPI and MA Craving: MA Cue $>$ Control Cue - NTX $>$ PLAC } \\
\hline Cluster I: Frontal cortex & & 1617 & & & & \\
\hline $\begin{array}{l}\text { Paracingulate gyrus/ } \\
\text { superior frontal gyrus }\end{array}$ & $\mathrm{R}$ & & 3.51 & 2 & 30 & 44 \\
\hline Precentral gyrus & $\mathrm{R}$ & & 3.44 & 40 & 6 & 30 \\
\hline Middle frontal gyrus & $\mathrm{R}$ & & 2.91 & 32 & 8 & 46 \\
\hline Paracingulate gyrus & L & & 2.89 & -4 & 20 & 48 \\
\hline
\end{tabular}

Results are from the psychophysiological interaction (PPI) analysis showing functional connectivity during methamphetamine cues processing

(Methamphetamine Cue > Control Cue contrast), using the precuneus as a functionally constrained, anatomically defined region of interest (see Methods). Analyses were whole-brain cluster-corrected at $Z>1.96, p<0.05$.

Note: $X, Y$, and $Z$ MNI coordinates indicate the location of peak voxel activation (or local maxima for subregions) within each cluster. R, right, L, left.

interaction with VTA $\kappa$-opioid receptors (Margolis et al, 2006), and by decreasing inhibition of excitatory output from the PFC to the dorsal striatum, which would, in turn, appear as enhanced functional connectivity between the VTA, PFC, and dorsal striatum. Given that inputs from the PFC to the dorsal striatum regulate the expression of reward-driven decision-making (Delgado et al, 2004; Haber et al, 2006), and that increased fronto-striatal connectivity is associated with greater frontal regulation over subcortical signals reflecting heightened reward sensitivity (Heatherton and Wagner, 2011), these results suggest greater frontal regulation of salience attribution was occurring during methamphetamine cue processing under opioid blockade (Goldstein and Volkow, 2011; Hare et al, 2009). Further, naltrexone may be modulating the visual corticostriatal loop, which includes connections from the ventromedial occipital cortex to the caudate specifically (Seger, 2013), and in turn may represent altered visual information processing of the methamphetamine cues during opioid blockade.

Importantly, naltrexone-moderated caudate functional connectivity during methamphetamine cues processing was found to correlate with self-reported tonic craving. Specifically, naltrexone was associated with stronger positive correlations between self-reported craving and caudate connectivity to prefrontal regions, as compared to placebo, potentially indicating greater recruitment of frontal involvement with dorsal striatal functioning during the experience of heightened subjective craving while under opioid blockade.

Naltrexone treatment was also associated with weaker precuneus functional connectivity to sensorimotor regions including the precentral and postcentral gyri during

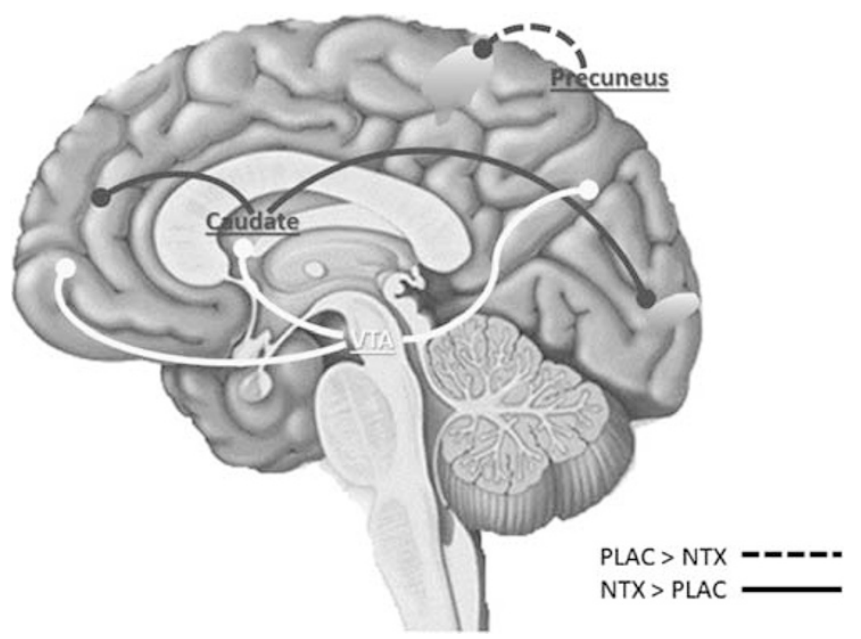

Figure 3 Schematic of the functional connectivity results. Yellow-orange blotches represent the areas where the naltrexone (NTX) condition was associated with reduced activation compared to the placebo (PLAC) condition (PLAC > NTX) within the Methamphetamine Cue > Control Cue contrast. Solid lines depict greater regional functional connectivity in the NTX $>$ PLAC contrast, and dashed lines greater regional functional connectivity in the PLAC>NTX contrast. No directionality of the connections is implied. A full color version of this figure is available at the Neuropsychopharmacology journal online.

methamphetamine cue processing. Interestingly, the precentral and postcentral gyri were also observed to be differentially affected by naltrexone in the main effect medication contrast, although the peak activation coordinates in the main effects analysis slightly differ from the connectivity results. The precuneus has strong, presumably GABAergic and glutamatergic cortical interconnections to the PFC, as well as connections to the PCC, superior parietal lobule, the dorsal premotor area, the supplementary motor area, and the ACC (for a review see Cavanna and Trimble, 2006). Functionally, the precuneus is putatively involved in selfcentered, mental imagery strategies and successful episodic memory retrieval (Cavanna and Trimble, 2006), both of which are likely to have a role in the phenomenology of craving. Thus, reduced functional connectivity between the precuneus and sensorimotor network during opioid blockade may reflect a reduced capacity for the integration of drug-cue information (Engelmann et al, 2012). Further, the opioid blockade appears to be facilitating greater frontal involvement with the precuneus as subjective craving increases, possibly reflecting the engagement of frontal regulatory mechanisms over mental imagery processing during the presentation of methamphetamine cues.

\section{Study Strengths and Limitations}

A major study strength is the within-subjects, placebocontrolled design that allowed for participants to serve as their own controls, addressing issues related to the comparison of medication effects across participants that may have differential structural brain changes due to varying histories of methamphetamine use (Berman et al, 2008). Another strength is the investigation of medication-induced alterations in CBF. Few fMRI studies effectively test medication-related $\mathrm{CBF}$ changes despite evidence of an interaction between basal physiologic and metabolic states 
and task-induced BOLD changes (Wang et al, 2011). The well-ascertained and clinically representative sample of individuals with methamphetamine use disorder also represents a strength, as it is often difficult to recruit individuals with severe drug addiction for clinical neuroscience research, yet these are precisely the individuals with which addiction science aims to help.

A limitation of the study is the relatively short duration of naltrexone treatment. It is possible that the 3-day dosing duration was not long enough for naltrexone to establish its full opioid blockade effect; thus the duration of dosing could potentially explain the absence of naltrexone-induced modulations of ventral striatum activation (and VTA activation at the whole-brain level of analysis) and measures of self-reported craving in the present study. However, the absence of naltrexone-related reductions in self-reported craving may also be related to the craving assessment procedure used (ie, phasic measurement of craving with a single item during scanning) as other studies have observed a benefit of using expanded item and response sets for the detection of drug craving correlations with neural measures of cue-reactivity (Courtney and Ray, 2014b). Further, previous studies have demonstrated complete hydromorphone blockade following a single dose of naltrexone (25 or 100 mg; Preston and Bigelow, 1993; Schuh et al, 1999) and others have observed reductions in alcohol and drug craving following 3-day dosing of naltrexone (Ray and Hutchison, 2007), suggesting that the measurement limitation theory may be a more viable explanation for the lack of naltrexone-related tonic craving reductions observed in the present study. Lastly, an analytic limitation of the present study is the use of atlas-based approaches to defining common ROIs used for all subjects. Greater sensitivity may have been achieved by segmenting the seed ROIs for each subject individually, which may be particularly relevant for detecting effects in smaller regions such as the NAcc.

\section{CONCLUSIONS}

The culmination of results from the present study suggests that the blockade of endogenous opioids may function to reduce the expression of drug cue salience by reducing sensorimotor processing and integration, and by engaging greater frontal regulation of salience attribution via pathways linking the dorsal striatum, VTA, and precuneus to frontal, striatal, and sensorimotor regions during cue processing. This is consistent with a previous report of naltrexone enhancing frontal engagement during a temporal discounting task in alcohol-dependent and control subjects (Boettiger et al, 2009), presumably reflecting greater fronto-striatal control over impulsive behavior (Courtney et al, 2013; Feil et al, 2010). Further, the development of pharmacotherapies that target and rescue deficient processes underlying the drug craving state, such as prefrontal cortical function, has been advanced as a promising strategy in treatment development (Sinha, 2013).

Importantly, at least some of these naltrexone-modulated frontal and sensorimotor functional pathways appear to subserve the experience of subjective methamphetamine craving. Successful self-regulation of craving is thought to represent a balance of bidirectional influence between prefrontal and subcortical regions involved in reward and reinforcement, such that self-regulatory failures occur whenever the balance tips in favor of subcortical areas (Heatherton and Wagner, 2011). To that end, naltrexone appears to be balancing prefrontal, subcortical, and parietal systems during methamphetamine cue processing, possibly via reductions of salience attribution in sensorimotor and reinforcement systems and by the enhancement of frontal control mechanisms.

In conclusion, these results provide the first evidence of changes in BOLD measures of methamphetamine cueinduced craving via blockade of endogenous opioids and suggest that naltrexone may be dampening the salience of the methamphetamine cues by reducing sensorimotor processing and integration, and by engaging greater frontal regulation of salience attribution during methamphetamine cue processing. These findings advance multiple neural mechanisms by which methamphetamine cue-induced craving may be instated, the knowledge of which may prove useful in the prediction of clinical outcomes and aid in the development of medications such as naltrexone for the treatment of various substance use disorders.

\section{FUNDING AND DISCLOSURE}

This study was supported by grants from the UCLA Training Program in Translational Neuroscience of Drug Abuse (T32 DA024635), the National Institute on Drug Abuse (F31 DA035604), Staglin IMHRO Center for Cognitive Neuroscience, and the UCLA Clinical \& Translational Research Center (CTSI Grant \#UL1TR000124). The authors declare no conflicts of interest.

\section{ACKNOWLEDGMENTS}

The authors would like to acknowledge Dr Nathasha Moallem, Katy Lunny, and Taylor Rohrbaugh for their contribution to data collection and data management, Dr Karen Miotto for serving as Study Physician, and Drs Danny JJ Wang and Kay Jann for their assistance with the cerebral blood flow analyses.

\section{REFERENCES}

Anggadiredja K, Sakimura K, Hiranita T, Yamamoto T (2004). Naltrexone attenuates cue- but not drug-induced methamphetamine seeking: a possible mechanism for the dissociation of primary and secondary reward. Brain Res 1021: 272-276.

Beck A, Wustenberg T, Genauck A, Wrase J, Schlagenhauf F, Smolka MN et al (2012). Effect of brain structure, brain function, and brain connectivity on relapse in alcohol-dependent patients. Arch Gen Psychiatry 69: 842-852.

Benjamin D, Grant ER, Pohorecky LA (1993). Naltrexone reverses ethanol-induced dopamine release in the nucleus accumbens in awake, freely moving rats. Brain Res 621: 137-140.

Berman S, O'Neill J, Fears S, Bartzokis G, London ED (2008). Abuse of amphetamines and structural abnormalities in the brain. Ann N Y Acad Sci 1141: 195-220.

Berridge KC, Robinson TE (2003). Parsing reward. Trends Neurosci 26: 507-513.

Boettiger CA, Kelley EA, Mitchell JM, D'Esposito M, Fields HL (2009). Now or later? An fMRI study of the effects of endogenous 
opioid blockade on a decision-making network. Pharmacol Biochem Behav 93: 291-299.

Carr DB, Sesack SR (2000). GABA-containing neurons in the rat ventral tegmental area project to the prefrontal cortex. Synapse 38: $114-123$.

Catafau AM, Etcheberrigaray A, Perez de los Cobos J, Estorch M, Guardia J, Flotats A et al (1999). Regional cerebral blood flow changes in chronic alcoholic patients induced by naltrexone challenge during detoxification. J Nucl Med 40: 19-24.

Cavanna AE, Trimble MR (2006). The precuneus: a review of its functional anatomy and behavioural correlates. Brain 129(Part 3): 564-583.

Courtney KE, Ghahremani DG, London ED, Ray LA (2014a). The association between cue-reactivity in the precuneus and level of dependence on nicotine and alcohol. Drug Alcohol Depend 141: 21-26.

Courtney KE, Ghahremani DG, Ray LA (2013). Fronto-striatal functional connectivity during response inhibition in alcohol dependence. Addict Biol 18: 593-604.

Courtney KE, Ray LA (2014b). Subjective responses to alcohol in the lab predict neural responses to alcohol cues. J Stud Alcohol Drugs 75: 124-135.

Courtney KE, Schacht JP, Hutchison K, Roche DJO, Ray LA (2016). Neural substrates of cue-reactivity: association with treatment outcomes and relapse. Addict Biol 21: 3-22.

de Wit H (1996). Priming effects with drugs and other reinforcers. Exp Clin Psychopharmacol 4: 5-10.

Delgado MR, Stenger VA, Fiez JA (2004). Motivation-dependent responses in the human caudate nucleus. Cereb Cortex 14: 1022-1030.

Emmerson PJ, Liu MR, Woods JH, Medzihradsky F (1994). Binding affinity and selectivity of opioids at mu, delta and kappa receptors in monkey brain membranes. J Pharmacol Exp Ther 271: 1630-1637.

Engelmann JM, Versace F, Robinson JD, Minnix JA, Lam CY, Cui Y et al (2012). Neural substrates of smoking cue reactivity: a metaanalysis of fMRI studies. Neuroimage 60: 252-262.

Everitt BJ, Robbins TW (2005). Neural systems of reinforcement for drug addiction: from actions to habits to compulsion. Nat Neurosci 8: 1481-1489.

Feil J, Sheppard D, Fitzgerald PB, Yucel M, Lubman DI, Bradshaw JL (2010). Addiction, compulsive drug seeking, and the role of frontostriatal mechanisms in regulating inhibitory control. Neurosci Biobehav Rev 35: 248-275.

Goldstein RZ, Volkow ND (2011). Dysfunction of the prefrontal cortex in addiction: neuroimaging findings and clinical implications. Nat Rev Neurosci 12: 652-669.

Haber SN, Kim K-S, Mailly P, Calzavara R (2006). Reward-related cortical inputs define a large striatal region in primates that interface with associative cortical connections, providing a substrate for incentive-based learning. J Neurosci 26: 8368-8376.

Hare TA, Camerer CF, Rangel A (2009). Self-control in decisionmaking involves modulation of the vmPFC valuation system. Science 324: 646-648.

Heatherton TF, Wagner DD (2011). Cognitive neuroscience of self-regulation failure. Trends Cogn Sci 15: 132-139.

Itzhak Y, Ali SF (2002). Behavioral consequences of methamphetamine-induced neurotoxicity in mice: relevance to the psychopathology of methamphetamine addiction. Ann N Y Acad Sci 965: 127-135.

Jayaram-Lindstrom N, Hammarberg A, Beck O, Franck J (2008). Naltrexone for the treatment of amphetamine dependence: a randomized, placebo-controlled trial. Am J Psychiatry 165: 1442-1448.

Kalivas PW, Volkow ND (2005). The neural basis of addiction: a pathology of motivation and choice. Am J Psychiatry 162: 1403-1413.
Kosten TR, Scanley BE, Tucker KA, Oliveto A, Prince C, Sinha R et al (2006). Cue-induced brain activity changes and relapse in cocaine-dependent patients. Neuropsychopharmacology 31: 644-650.

Lee YK, Park SW, Kim YK, Kim DJ, Jeong J, Myrick $\mathrm{H}$ et al (2005). Effects of naltrexone on the ethanol-induced changes in the rat central dopaminergic system. Alcohol Alcohol 40: $297-301$.

Lukas SE, Lowen SB, Lindsey KP, Conn N, Tartarini W, Rodolico J et al (2013). Extended-release naltrexone (XR-NTX) attenuates brain responses to alcohol cues in alcohol-dependent volunteers: a bold FMRI study. Neuroimage 78: 176-185.

Margolis EB, Lock H, Chefer VI, Shippenberg TS, Hjelmstad GO, Fields HL (2006). Kappa opioids selectively control dopaminergic neurons projecting to the prefrontal cortex. Proc Natl Acad Sci U S A 103: 2938-2942.

Miranda R, Ray L, Blanchard A, Reynolds EK, Monti PM, Chun T et al (2014). Effects of naltrexone on adolescent alcohol cue reactivity and sensitivity: an initial randomized trial. Addict Biol 19: 941-954.

Monti PM, Rohsenow DJ, Hutchison KE, Swift RM, Mueller TI, Colby SM et al (1999). Naltrexone's effect on cue-elicited craving among alcoholics in treatment. Alcohol Clin Exp Res 23: 1386-1394.

Murty VP, Shermohammed M, Smith DV, Carter RM, Huettel SA, Adcock RA (2014). Resting state networks distinguish human ventral tegmental area from substantia nigra. Neuroimage 100: 580-589.

O'Reilly JX, Woolrich MW, Behrens TE, Smith SM, Johansen-Berg H (2012). Tools of the trade: psychophysiological interactions and functional connectivity. Soc Cogn Affect Neurosci 7: 604-609.

Preston KL, Bigelow GE (1993). Differential naltrexone antagonism of hydromorphone and pentazocine effects in human volunteers. J Pharmacol Exp Ther 264: 813-823.

Ray LA, Bujarski S, Courtney KE, Moallem NR, Lunny K, Roche D et al (2015a). The effects of naltrexone on subjective response to methamphetamine in a clinical sample: a double-blind, placebo-controlled laboratory study. Neuropsychopharmacology 40: 2347-2356.

Ray LA, Courtney KE, Ghahremani DG, Miotto K, Brody A, London ED (2015b). Varenicline, naltrexone, and their combination for heavy-drinking smokers: preliminary neuroimaging findings. Am J Drug Alcohol Abuse 41: 35-44.

Ray LA, Courtney KE, Roche D, Miotto K (in press). Application of functional neuroimaging to the treatment of addiction: focus on pharmacotherapies. In: Filbey F, Feldstein-Ewing SW, Witkiewitz K (eds). Neuroimaging and Psychosocial Addiction Treatment: sAn Integrative Guide for Researchers and Clinicians. Palgrave: New York, NY.

Ray LA, Hutchison KE (2007). Effects of naltrexone on alcohol sensitivity and genetic moderators of medication response: a doubleblind placebo-controlled study. Arch Gen Psychiatry 64: 1069-1077.

Ray LA, Hutchison KE, MacKillop J, Miranda R Jr, Audette A, Swift $\mathrm{R}$ et al (2008). Effects of naltrexone during the descending limb of the blood alcohol curve. Am J Addict 17: 257-264.

Robinson TE, Berridge KC (2001). Incentive-sensitization and addiction. Addiction 96: 103-114.

Schacht JP, Anton RF, Myrick H (2013a). Functional neuroimaging studies of alcohol cue reactivity: a quantitative meta-analysis and systematic review. Addict Biol 18: 121-133.

Schacht JP, Anton RF, Randall PK, Li X, Henderson S, Myrick H (2013b). Effects of a GABA-ergic medication combination and initial alcohol withdrawal severity on cue-elicited brain activation among treatment-seeking alcoholics. Psychopharmacology (Berl) 227: 627-637.

Schuh KJ, Walsh SL, Stitzer ML (1999). Onset, magnitude and duration of opioid blockade produced by buprenorphine and naltrexone in humans. Psychopharmacology (Berl) 145: 162-174. 
Seger CA (2013). The visual corticostriatal loop through the tail of the caudate: circuitry and function. Front Syst Neurosci 7: 104.

Sinha R (2013). The clinical neurobiology of drug craving. Curr Opin Neurobiol 23: 649-654.

Syed YY, Keating GM (2013). Extended-release intramuscular naltrexone (VIVITROL(R)): a review of its use in the prevention of relapse to opioid dependence in detoxified patients. CNS Drugs 27: 851-861.

Tien LT, Ho IK, Loh HH, Ma T (2007). Role of mu-opioid receptor in modulation of preproenkephalin mRNA expression and opioid and dopamine receptor binding in methamphetamine-sensitized mice. J Neurosci Res 85: 673-680.

Toll L, Berzetei-Gurske IP, Polgar WE, Brandt SR, Adapa ID, Rodriguez L et al (1998). Standard binding and functional assays related to medications development division testing for potential cocaine and opiate narcotic treatment medications. NIDA Res Monogr 178: 440-466.
Volkow ND, Wang GJ, Telang F, Fowler JS, Logan J, Childress AR et al (2006). Cocaine cues and dopamine in dorsal striatum: mechanism of craving in cocaine addiction. J Neurosci 26: 6583-6588.

Vollstadt-Klein S, Wichert S, Rabinstein J, Buhler M, Klein O, Ende $\mathrm{G}$ et al (2010). Initial, habitual and compulsive alcohol use is characterized by a shift of cue processing from ventral to dorsal striatum. Addiction 105: 1741-1749.

Wang DJ, Chen Y, Fernandez-Seara MA, Detre JA (2011). Potentials and challenges for arterial spin labeling in pharmacological magnetic resonance imaging. J Pharmacol Exp Ther 337: 359-366.

Worsley KJ (2001). Statistical analysis of activation images. In: Jezzard P, Matthews PM, Smith SM (eds). Functional MRI: An Introduction to Methods. Oxford University Press: New York, NY. Zubieta JK, Gorelick DA, Stauffer R, Ravert HT, Dannals RF, Frost JJ (1996). Increased mu opioid receptor binding detected by PET in cocaine-dependent men is associated with cocaine craving. Nat Med 2: 1225-1229.

Supplementary Information accompanies the paper on the Neuropsychopharmacology website (http://www.nature.com/npp) 